\title{
Contribuição ao estudo do magmatismo Paleo e Mesoproterozóico do SW do Craton Amazônico através da aplicação de isótopos estáveis de $0, \mathrm{H}$ e S
}

\author{
Mauro Cesar GERALDES ${ }^{1}$, Wilson TEIXEIRA², Claudia Sayão VALLADARES³
}

\begin{abstract}
RESUMO
Os isótopos estáveis de $\mathrm{O}, \mathrm{H}$ e S foram utilizados para investigar a origem das rochas magmáticas nos Terrenos Jauru e Pontes e Lacerda do SW do Craton Amazônico, estado de Mato Grosso, Brasil. No Terreno Jauru as rochas granitóides do Greenstone belt Alto Jauru e da Suíte Cachoeirinha apresentam valores de $\delta^{18} \mathrm{O}$ entre $+9,0 \%$ e $+6,3 \%$ o que indicam derivação a partir de magmas juvenis. $\mathrm{Na}$ Suíte Intrusiva Rio Branco valores de $\delta^{18} \mathrm{O}$ para rochas básicas estão entre $+5,4 \%$ e $+5,8 \%$ e para rochas félsicas entre $+8,7 \%$ e $+9,0 \%$; rochas intermediárias apresentam valores entre $+7,3 \%$ e $+8,3 \%$. Os valores mais baixos de $\delta^{18} \mathrm{O}$, obtidos nas rochas básicas, são compatíveis com derivação mantélica, porém as rochas félsicas apresentam valores de $\delta^{18} \mathrm{O}$ compatíveis com origem crustais. Análises de isótopos estáveis de $\mathrm{H}$ (rocha total) forneceram valores de $\delta \mathrm{D}$ entre $-83 \% 0$ e -92\%o, diferente das assinaturas de rochas metamórficas e de águas meteóricas. Resultados em sulfetos para isótopos estáveis de $S$ em rochas básicas e intermediárias desta suíte apresentam valores de $\delta^{34} S$ coerentes com uma fonte mantélica (entre + $0,7 \%$ e $+3,8 \%$ ) , enquanto os valores de $\delta^{34} S$ (entre $+5,2 \%$ e $+6,1 \%$ ) obtidos nas rochas félsicas sugerem participação crustal na sua gênese. Na Suíte Santa Helena (Terreno Pontes e Lacerda) os resultados obtidos para $\delta^{18} \mathrm{O}$ se agrupam entre $+4,4 \%$ e $+8,9 \%$ indicando uma origem mantélica. O presente estudo confirma a importância da aplicação de isótopos estáveis para a compreensão de processos magmáticos e evolução crustal.
\end{abstract}

PALAVRAS-ChAVE: Magmatismo, Isótopos de Oxigênio, Isótopos de Hidrogênio, Isótopos de Enxofre, Craton Amazônico

\section{Contribution to the study of the Paleo and Mesoproterozoic magmatism of the SW Amazonian craton based on stable isotopes of $\mathrm{O}, \mathrm{H}$ and $\mathrm{S}$}

\begin{abstract}
Stable isotopes of $\mathrm{O}, \mathrm{H}$ and $\mathrm{S}$ were used to investigate the origin of magmatic rocks of the Jauru and the Pontes e Lacerda Terrains, SW portion of the Amazonian craton, Mato Grosso state, Brazil. Granitic rocks of the Alto Jauru Greenstone belt and Cachoeirinha Suite, Jauru terrain, present $\delta^{18} \mathrm{O}$ values between $+9.0 \%$ and $+6.3 \%$, indicative of derivation from juvenile magmas. The Rio Branco Intrusive Suite basic and felsic rocks' $\delta^{18} \mathrm{O}$ values fall, respectively, within $+5.4 \% 0$ to $+5.8 \%$ and $+8.7 \%$ o to $+9.0 \%$ ranges; the intermediate rocks present $\delta^{18} \mathrm{O}$ between $+7.3 \%$ and $+8.3 \%$. The lower values of $\delta^{18} \mathrm{O}$, obtained from basic rocks, are compatible with a mantle source, however the felsic rocks present $\delta^{18} \mathrm{O}$ values indicative of crustal source. The stable isotopes of hydrogen yielded $\delta \mathrm{D}$ values between $-83 \%$ and $-92 \%$, different from the $\delta \mathrm{D}$ signatures of metamorphic rocks and rain water. Sulphur isotopes in sulfides from basic and intermediate rocks of the Rio Branco suite presented $\delta^{34} S$ values coherent with a mantle source (between $+0.7 \%$ ond $+3.8 \%$ ), whereas $\delta^{34} S$ values between $+5.2 \%$ o and $+6.1 \%$, obtained from the felsic rocks, suggest crustal participation in their genesis. For the Santa Helena Suite (Pontes e Lacerda Terrain) the $\delta^{18} \mathrm{O}$ values fall between $+4.4 \%$ and $+8.9 \%$. The present study confirms the advantages of using stable isotopes to understand magmatic processes and crustal evolution.
\end{abstract}

KEY WORDS: Magmatism, Oxygen Isotopes, Hydrogen Isotopes, Sulphur Isotopes, Amazonian craton.

\footnotetext{
1 Faculdade de Geologia, UERJ, Rua São Francisco Xavier 524, Maracanã. CEP 20550-013 Rio de Janeiro (RJ), Brasil. e-mail: geraldes@uerj.br

2 Instituto de Geociências, USP, Rua do Lago 562, Cidade Universitária. CEP 05508-900 São Paulo (SP), Brasil. e-mail: wteixeir@usp.br

${ }^{3}$ Faculdade de Geologia, UERJ, Rua São Francisco Xavier 524, Maracanã. CEP 20550-013 Rio de Janeiro (RJ), Brasil. e-mail: vallada@uerj.br
} 


\section{INTRODUÇÃO}

Os isótopos estáveis constituem uma ferramenta efetiva na distinção entre rochas derivadas do manto daquelas formadas a partir da crosta continental. O estudo de isótopos de oxigênio em conjunção com os isótopos de hidrogênio e enxofre tem se mostrado uma ferramenta poderosa na investigação de processos petrológicos em função de assinaturas isotópicas específicas apresentadas para cada ambiente geológico. Em relação aos isótopos de oxigênio, a crosta é enriquecida em $\delta^{18} \mathrm{O}$ em relação ao manto em conseqüência da interação da crosta com a hidrosfera (Forester \& Taylor, 1977; O’Neil et al., 1977; Sial, 1984; Sial et al., 1996; Harris et al., 1997). Em adição, os isótopos de $\mathrm{H}$ e $\mathrm{S}$ definem assinaturas que podem ser utilizadas em estudos petrogenéticos e como traçadores da origem das soluções hidrotermais (Wenner \& Taylor, 1976; Nabelek et al., 1983; Sial \& Ferreira, 1990).

A maioria das rochas graníticas (O’Neil \& Taylor, 1967; Taylor, 1980; Taylor, 1987; Clayton, Goldsmith \& Mayeda, 1989; Ryerson \& McKeegan, 1994; Palin, Epstein \& Stolper, 1996), metamórficas (Agrinier et al., 1985; Nabelek, 1991; Van Wyck et al., 1966) e sedimentares (Claypool et al., 1980; Vanko et al., 1992; Rollinson, 1993) é enriquecida em $\delta^{18} \mathrm{O}$ relativo aos valores do manto $\left(\delta^{18} \mathrm{O}=5,75 \pm 0,5 \%\right.$ ) enquanto a água do mar (que pode modificar as assinaturas isotópicas de rochas formadas no assoalho oceânico) e as águas de soluçôes metamórficas são empobrecidas. A aplicação de isótopos estáveis também tem sido de grande utilidade para a caracterização de modelos genéticos de depósitos minerais, como exemplificado nos trabalhos de Kanehiro et al. (1973), Golding \& Wilson (1983), Spooner et al. (1985), Goodz et al. (1986), Iyer et al., (1992), Martarelli et al. (1995). O objetivo deste trabalho é o estudo de isótopos estáveis de $\mathrm{O}, \mathrm{H}$ e $\mathrm{S}$ dos granitóides da porção SW do estado do Mato Grosso. A abordagem deste artigo tem relevância para o entendimento da evolução magmática dos corpos ígneos da região enfocada, gerados em eventos de acreção crustal durante o Paleoproterozóico e o Mesoproterozóico que vieram a compor significativa fração do SW do Cráton Amazônico.

\section{A GEOLOGIA DO SW DO CRATON AMAZÔNICO}

A integração das informaçôes disponíveis na literatura a respeito da evolução geológica da região permitiu delinear três terrenos com características distintas considerando a associação litológica, dados litogeoquímicos, dados isotópicos, dados de campo e arranjo estrutural (Tassinari et al., 2000; Geraldes et al., 2001). Estes terrenos (Jauru, Pontes e Lacerda e Rio Alegre), têm seus limites definidos por grandes lineamentos estruturais confirmados por dados geofísicos (Menezes et al., 1993).

O Terreno Jauru (Figura 1a) agrupa as unidades (greenstone belt Alto Jauru, Suíte Cachoeirinha e Suíte Intrusiva Rio
Branco) que ocorrem no lado leste do lineamento TaquaruçuJundiuvira-Lucialva (Monteiro et al., 1986) recobertas ao norte por sedimentos do Grupo Parecis (Saes et al., 1984). Tonalitos, vulcânicas ácidas e gnaisses do greenstone belt Alto Jauru foram analisados pelo método $\mathrm{U}-\mathrm{Pb}$ em zircóes e apresentaram idades de 1790 a 1750 Ma. Análises químicas de rocha total indicam características calcioalcalinas e dados isotópicos Sm-Nd ( $\mathrm{T}_{\mathrm{DM}}$ entre 2000 e $1800 \mathrm{Ma}$ e $\varepsilon_{\mathrm{Nd}(\mathrm{t})}$ entre +3 e +2 ) reforçam as características juvenis para esta unidade (Geraldes et al., 2001). Em adição, rochas intrusivas da Suíte Cachoeirinha (com composição entre granito e tonalito) apresentam idades U-Pb em zircóes entre $1585 \mathrm{Ma}$ e 1536 Ma. O estudo químico destas rochas indica trend calcioalcalino e resultados isotópico Sm-Nd ( $\mathrm{T}_{\mathrm{DM}}$ entre 2047 e $1743 \mathrm{Ma}$ e $\varepsilon_{\mathrm{Nd}(\mathrm{t})}$ entre $\left.+3,7 \mathrm{e}-1,3\right)$ sugerem ainda que a sua formação ocorreu em arco magmático desenvolvido na margem continental pré-existente, com significativo retrabalhamento de crosta mais antiga durante a geração destes corpos plutônicos (Ruiz et al., 2004). A Suíte Intrusiva Rio Branco representa parte de uma associação bimodal na região de Jauru-Araputanga (Terreno Jauru) com idades U-Pb entre 1460 Ma e 1420 Ma. Rochas de composição básica a félsica e estruturas rapakivi indicam mistura de magmas, o que é também sugerido pela composição bimodal identificada nos estudos litogeoquímicos desta unidade. Dados de isótopos de Sm-Nd para as unidades básicas $\left(\mathrm{T}_{\mathrm{DM}}\right.$ entre 1800 e $1700 \mathrm{Ma}$ e $\varepsilon_{\mathrm{Nd}(\mathrm{t})}$ entre $\left.+1,9 \mathrm{e}+1,2\right)$ e para as unidades félsicas $\left(\mathrm{T}_{\mathrm{DM}}\right.$ entre 1700 e $1600 \mathrm{Ma}$ e $\varepsilon_{\mathrm{Nd}(\mathrm{t})}$ entre $+0,9$ e $\left.-0,1\right)$ sugerem que os protólitos destas rochas têm origem mantélica e da base da crosta, respectivamente (Geraldes et al., 2004). Em adição, observam-se intensos processos de sericitização nas rochas félsicas e cloritização e saussiritização nas rochas básicas ao longo de toda a unidade.

O Terreno Pontes e Lacerda (Figura 1b) localiza-se a oeste do lineamento Taquaruçu-Jundiuvira-Lucialva, sendo limitado ao oeste pela faixa de sedimentos deformados do Grupo Aguapeí (Souza \& Hildred, 1980). Este terreno é composto por rochas da Suíte Santa Helena além de granitos anorogênicos não foliados, como o Guapé e o São Domingos com idade U-Pb de $\sim$ 1,0 Ga (Menezes, Lopes \& Bezerra, 1993). As rochas da Suíte Santa Helena apresentam idades U-Pb em zircão entre 1,45 Ga e 1,42 Ga e $\mathrm{T}_{\mathrm{DM}}$ entre 1,70 Ga e 1,50 Ga (com $\varepsilon_{\mathrm{Nd}(\mathrm{t})}$ entre $+4,1$ e +2,6). São rochas de composição granítica a tonalítica, com quimismo calcioalcalino, sugerindo formação em ambiente de arco magmático distal à margem continental pré-existente (Terreno Jauru) a qual teve participação subordinada na geração do plutonismo (Tassinari et al., 2000).

O Terreno Rio Alegre $(1,51 \mathrm{Ga}$ a $1,48 \mathrm{Ga})$ e o Terreno Pontes e Lacerda são limitados pelo Lineamento Rio Alegre (LRA), definido como uma zona de cisalhamento formada 


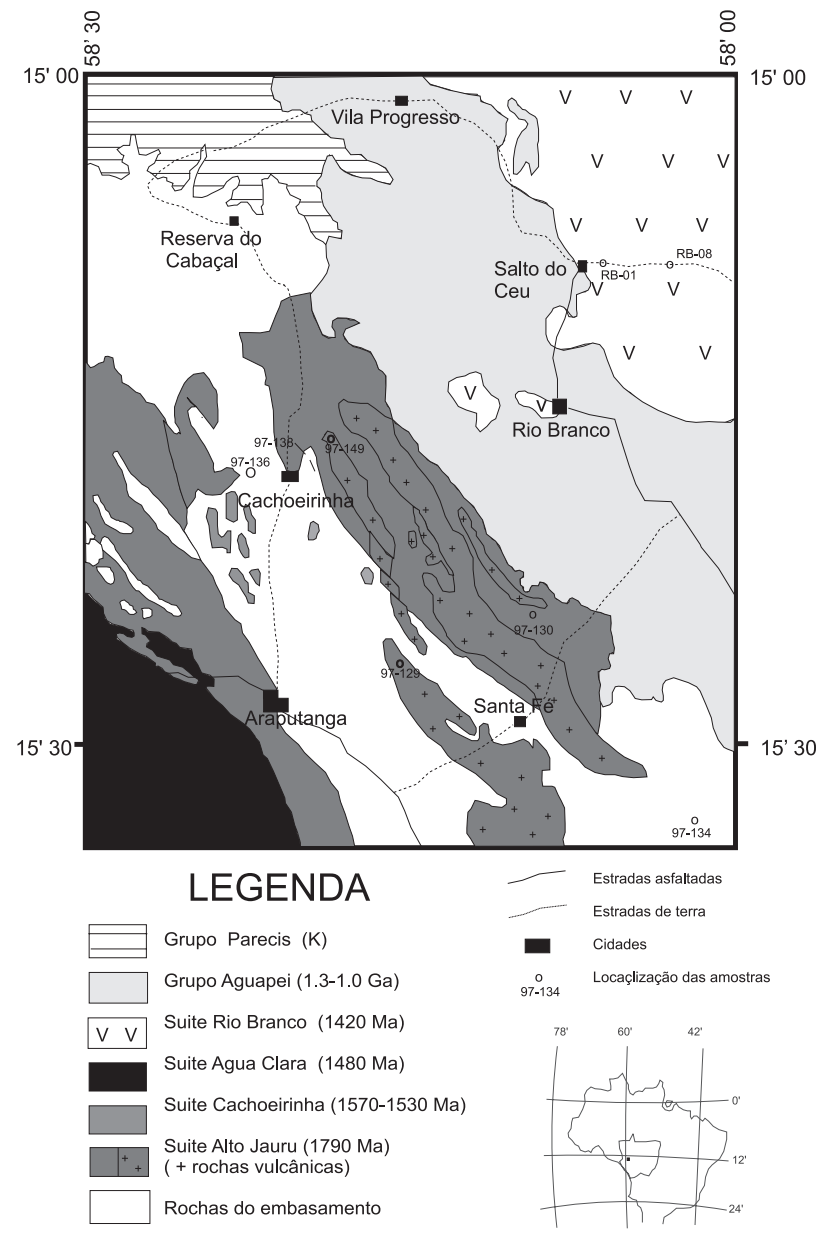

Figura 1a - Mapa geológico simplificado da região de Jauru-Araputanga.

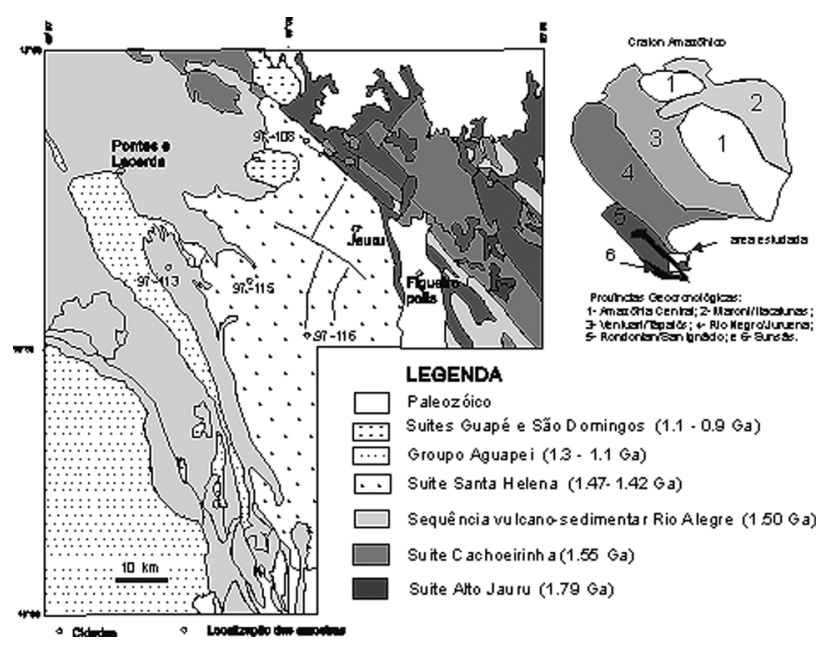

Figura 1b - Mapa geológico simplificado da região de Pontes e Lacerda. durante o Evento Aguapeí. Este lineamento apresenta anomalias gravimétricas e cintilométricas e foi interpretado como uma zona de sutura reativada que representaria o depocentro da bacia Aguapeí (Menezes et al., 1993). O Terreno Rio Alegre é constituído por rochas vulcanossedimentares metamorfisadas na fácies xisto-verde, com características distintas dos granitos típicos do Suíte Santa Helena, que predominam no Terreno Pontes e Lacerda. As rochas deformadas do Grupo Aguapeí estão presentes tanto no Terreno Pontes e Lacerda como no Terreno Rio Alegre.

\section{MATERIAL E MÉTODOS}

As análises dos isótopos estáveis de $\mathrm{O}\left({ }^{18} \mathrm{O}\right.$ e $\left.{ }^{16} \mathrm{O}\right), \mathrm{S}\left({ }^{34} \mathrm{~S} /{ }^{32} \mathrm{~S}\right)$ e $\mathrm{H}$ (deutério $\mathrm{e}^{1} \mathrm{H}$ ) foram realizadas no laboratório ACTLABS no Canadá em amostras de rocha total pulverizadas (200 mesh) estudadas pelos métodos $\mathrm{U}-\mathrm{Pb}, \mathrm{Sm}-\mathrm{Nd}$ e geoquímica de rocha total (Geraldes et al., 2001). A extração de oxigênio de rocha total foi realizada por reação com pentafluoreto de bromo por um período de 12 horas e temperatura de $600^{\circ} \mathrm{C}$, segundo procedimentos descritos por Clayton \& Mayeda (1963). As amostras foram então convertidas para $\mathrm{CO}_{2}$ por reação com um bastão de carbono e este gás foi então analisado por espectrometria de massa. As análises de isótopos de $\mathrm{O}$ foram realizadas em 21 amostras, distribuídas nos terrenos Jauru e Pontes e Lacerda. Para o Terreno Jauru foram realizadas 3 análises para as rochas do greenstone belt Alto Jauru, 3 para as rochas da Suíte Cachoeirinha e 8 na Suíte Intrusiva Rio Branco. Para o Terreno Pontes e Lacerda foram realizadas 7 análises na Suíte Santa Helena.

Análises de $S$ (segundo o procedimento descrito por Iyer $e t$ al., 1992) foram realizadas através da reação KIBA (com ácido fosfórico estanhoso) em piritas separadas das amostras da suíte Rio Branco. O enxofre é extraído como sulfeto de hidrogênio e posteriormente transformado em sulfeto de prata. Este último é submetido à combustão na presença de $\mathrm{Cu}_{2} \mathrm{O}$ a $800^{\circ} \mathrm{C}$, liberando o $\mathrm{SO}_{2}$ e outros compostos. Este gás é purificado e o enxofre é analisado em espectrômetro de massa. As amostras da suíte Rio Branco foram processadas também para a separação de micas com o objetivo de se analisar isótopos de $\mathrm{H}$. O hidrogênio é analisado segundo procedimento de $\mathrm{O}^{\prime} \mathrm{Neil}$ (1979), de forma que a água é coletada por fusão da amostra e as razões isotópicas de $\mathrm{H} / \mathrm{D}$ medidas em espectrômetro de massa. Análises de $\mathrm{S}$ e $\mathrm{H}$ foram realizadas nas amostras da Suíte Intrusiva Rio Branco, resultando em 6 análises de isótopos de $\mathrm{H}$ (micas) e 6 análises de isótopos de $\mathrm{S}$ (piritas). 


\section{RESULTADOS E DISCUSSÃO}

\section{RESULTADOS ISOTÓPICOS}

\section{TERRENO JAURU}

Seis amostras de rochas granitóides intrusivas do Terreno Jauru foram analisadas para isótopos de oxigênio, são estas: Tonalito Cabaçal, Gnaisse Aliança, Gnaisse Rosa, pertencentes ao greenstone belt Alto Jauru; e Granito Alvorada, Granodiorito Água Clara e Granito Cachoeirinha da Suíte Cachoeirinha. Os resultados de ${ }^{18} \mathrm{O} /{ }^{16} \mathrm{O}$ foram normalizados pelo standard SMOW e estão apresentados na Tabela 1. A localização das amostras encontra-se na Figura 1A.

Os dados isotópicos $\delta^{18} \mathrm{O}$ obtidos para os tonalitos e gnaisses do greentone belt Alto Jauru (Tabela 1) indicam uma variação de valores entre $+6,3 \%$ e $+6,6 \%$. Os valores obtidos foram: para o Tonalito Cabaçal $\delta^{18} \mathrm{O}=+6,6 \%$, para o Gnaisse Aliança $\delta^{18} \mathrm{O}=+6,3 \%$, e para o Gnaisse Rosa $\delta^{18} \mathrm{O}=+6,3 \%$. Estes valores são coerentes com os dados de $\mathrm{Sm}-\mathrm{Nd}$ para as mesmas amostras ( $\mathrm{T}_{\mathrm{DM}}$ entre 2000 e $1800 \mathrm{Ma}$ e $\varepsilon_{\mathrm{Nd}(\mathrm{t})}$ entre +3 e +2 , ver Tabela 1), que são característicos de suítes calcioalcalinas geradas em arcos magmáticos. De acordo com Taylor (1974), valores de $\delta^{18} \mathrm{O}$ entre $+6,0 \%$ e e $+8,0 \%$ indicam origem mantélica.

A Suíte Cachoeirinha é representada pelas amostras do Granito Alvorada $\left(\delta^{18} \mathrm{O}=+6,3 \%\right)$, Granodiorito Água Clara $\left(\delta^{18} \mathrm{O}=+9,0 \%\right.$ o $)$ e do Granito Cachoeirinha $\left(\delta^{18} \mathrm{O}=+7,2 \%\right.$ ) (Tabela 1). $\mathrm{O}$ alto valor de $\delta^{18} \mathrm{O}$ para a amostra $97-136$ (Granodiorito Água Clara) não é coerente com o valor $\mathrm{de} \boldsymbol{E}_{\mathrm{Nd}(\mathrm{t})}=$ $+1,7$ que indica uma origem juvenil para este corpo. Os estudos petrográficos e geoquímicos não apontaram características que explicassem o alto valor de $\delta^{18} \mathrm{O}$ para esta amostra. Em adição, outros exemplos de rochas mantoderivadas com valores de $\delta^{18} \mathrm{O}$ maiores de $8 \%$ são reportados por Baumgartner $\&$

Tabela 1 - Valores de $\delta 0^{18}$ para as rochas granitóides do terreno Jauru. As razões ${ }^{18} 0 /{ }^{16} 0$ foram normalizadas pelo standard SMOW (standard mean ocean water). Valores de U-Pb e Sm-Nd (Geraldes et al. 2001).

\begin{tabular}{|c|c|c|c|c|c|c|}
\hline Amostra & Unidade & Descrição & $\delta 0^{18}$ & $\varepsilon_{\mathrm{Nd}(t)}$ & $\mathrm{T}_{\mathrm{DM}}(\mathrm{Ga})$ & $\mathrm{U}-\mathrm{Pb}(\mathrm{Ma})$ \\
\hline $97-130$ & $\begin{array}{l}\text { Greenstone } \\
\text { belt Alto } \\
\text { Jauru }\end{array}$ & $\begin{array}{l}\text { Tonalito } \\
\text { Cabaçal }\end{array}$ & $+6,6$ & $+2,6$ & 1,85 & $\begin{array}{l}1767 \pm \\
24\end{array}$ \\
\hline $97-149$ & $\begin{array}{l}\text { Greenstone } \\
\text { belt Alto } \\
\text { Jauru }\end{array}$ & $\begin{array}{l}\text { Gnaisse } \\
\text { Aliança }\end{array}$ & $+6,3$ & $+2,4$ & 1,77 & $\begin{array}{l}1746 \pm \\
20\end{array}$ \\
\hline $97-134$ & $\begin{array}{l}\text { Greenstone } \\
\text { belt Alto } \\
\text { Jauru }\end{array}$ & $\begin{array}{l}\text { Gnaisse } \\
\text { Rosa }\end{array}$ & $+6,3$ & $+2,2$ & 1,93 & $\begin{array}{l}1795 \pm \\
10\end{array}$ \\
\hline $97-129$ & $\begin{array}{l}\text { Suíte } \\
\text { Cachoeirinha }\end{array}$ & $\begin{array}{l}\text { Granito } \\
\text { Alvorada }\end{array}$ & $+6,3$ & $+0,9$ & 1,78 & $\begin{array}{l}1522 \pm \\
12\end{array}$ \\
\hline $97-136$ & $\begin{array}{l}\text { Suíte } \\
\text { Cachoeirinha }\end{array}$ & $\begin{array}{l}\text { Granodiorito } \\
\text { Água Clara }\end{array}$ & $+9,0$ & $+1,7$ & 1,77 & $\begin{array}{l}1485 \pm \\
04\end{array}$ \\
\hline $97-138$ & $\begin{array}{l}\text { Suíte } \\
\text { Cachoeirinha }\end{array}$ & $\begin{array}{l}\text { Granito } \\
\text { Cachoeirinha }\end{array}$ & $+7,2$ & $+0,5$ & 1,75 & $\begin{array}{l}1536 \pm \\
06\end{array}$ \\
\hline
\end{tabular}

Rumble (1988). Segundo estes autores, o aumento dos valores de $\delta^{18} \mathrm{O}$ pode ocorrer por processos de transporte de isótopos posteriormente à cristalização dos minerais, como discutido por Eiler et al. (1992), e Roedder (1992). Desta forma, com exceção do valor obtido para o Granodiorito Água Clara (+9,0\%o), os dados obtidos permitem concluir que os granitóides analisados no Terreno Jauru (Figura 2) foram derivados de magmas juvenis e não apresentam indícios de processos ocorridos a baixas temperaturas.

Por outro lado, os estudos isotópicos (U-Pb e Sm-Nd; Ruiz et al., 2004; Tassinari et al., 2000) reportados para as rochas da Suíte Cachoeirinha, incluindo amostras não analisadas para isótopos de $\mathrm{O}$, indicam geração a partir de crosta oceânica em subducção que provocou a hidratação e a fusão parcial do manto. Desta forma, pode-se sugerir a participação de sedimentos oriundos da placa em subducção e/ou retrabalhamento de crosta mais antiga na geração dos protólitos destas rochas, como nos casos de rochas com valores de $\delta^{18} \mathrm{O}$ de $+8 \%$ o e $+9 \%$, $\mathrm{T}_{\mathrm{DM}}$ de $-2,0$ Ga e $\varepsilon_{\mathrm{Nd}}$ entre $+1,7$ e $-1,3$, resultando em variaçôes composicionais e isotópicas $\left(\varepsilon_{\mathrm{Nd}(\mathrm{t})}\right.$ negativos) nos magmas que deram origem à suíte calcioalcalina de idade Mesoproterozóica do Terreno Jauru.

Oito amostras da Suíte Intrusiva Rio Branco (rocha total) foram analisadas para isótopos estáveis de oxigênio, cujos resultados foram normalizados pelo standard SMOW $\left({ }^{18} \mathrm{O} /{ }^{16} \mathrm{O}=0,0039948\right)$ (Tabela 2, Figura 3). Desta forma, os valores de $\delta^{18} \mathrm{O}$ obtidos variam de $+5,4 \%$ a $+5,8 \%$ o (nas rochas básicas); rochas intermediárias apresentam valores entre $+7,3 \%$ o e $+8,3 \%$; e valores entre $7,3 \%$ e e $+9,0 \%$ s são observados em rochas ácidas. Portanto, as rochas do primeiro

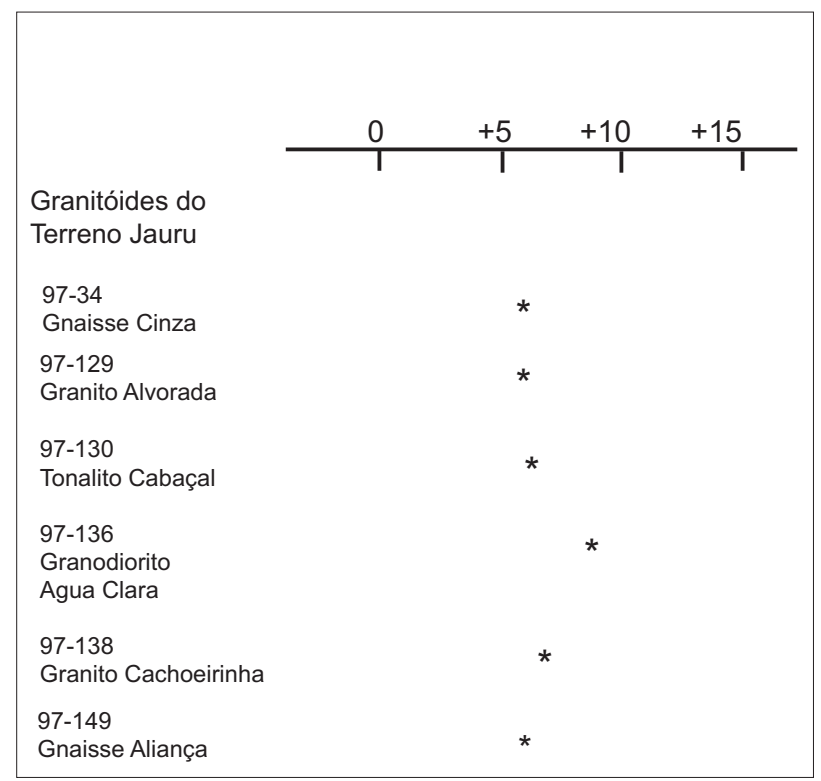

Figura 2 - Valores de $\delta^{18} 0$ para as rochas intrusivas Mesoproterozóicas do Terreno Jauru. 
grupo não apresentam influência crustal, diferentemente das rochas do segundo e do terceiro grupo. No segundo grupo, as rochas com valores de $\delta^{18} \mathrm{O}$ entre $+7,3 \%$ e e $+8,3 \%$ o podem ter sido originadas por mistura de magmas e mingling. Os resultados obtidos para rochas básicas (gabros e basaltos) são compatíveis com as variações de $\delta^{18} \mathrm{O}$ descritos por Taylor (1974) e de sienitos e traquitos como reportado por Sial \& Ferreira (1990). O primeiro grupo apresenta resultados equivalentes com valores de $\delta^{18} \mathrm{O}$ próximos de $5,5 \%$, normais em rochas manto-derivadas (gabros e basaltos).

Os resultados das análises de isótopos estáveis de $\mathrm{H}$ (rocha total) para as mesmas amostras da Suíte Intrusiva Rio Branco indicam valores de $\delta \mathrm{D}$ entre $-83 \%$ e $-92 \%$. Os resultados de isótopos de $\mathrm{H}$ das amostras analisadas apresentam assinaturas distintas da assinatura isotópica atual de águas marinhas (SMOW apresenta $\delta \mathrm{D}=0)$. Esta diferença indica a inexistência de contaminação atual por águas superficiais.

Em adição, estes resultados, quando lançados no diagrama $\delta \mathrm{D}$ versus $\delta^{18} \mathrm{O}$ (Figura 4) não apóiam a participação de águas metamórficas na formação das rochas estudadas. Exemplos de rochas ígneas alteradas por água marinha apresentam forte assinatura isotópica de $\mathrm{H}$, conforme caso reportado por Vanko et al. (1992) em rochas basálticas da Cadeia Meso-Atlântica.

Os resultados de isótopos estáveis de $S$ (em piritas) nas mesmas amostras indicam uma variação dos valores de $\delta^{34} S$ entre $+0,7 \%$ e e $+6,1 \%$. As relações texturais dos grãos de sulfetos indicam origem magmática, o que permite sugerir que os resultados isotópicos de $S$ indicam a composição

Tabela 2 - Dados de isótopos de 0, He S para as rochas da Suíte Intrusiva Rio Branco. Valores de U-Pb e Sm-Nd (Geraldes et al. 2004).

\begin{tabular}{llcccccc}
\hline Amostra & Tipo de rocha & $\delta^{18} 0$ & $\delta \mathrm{D}$ & $\delta \mathrm{S}$ & $\mathrm{T}_{\mathrm{DM}}(\mathrm{Ga})$ & $\varepsilon_{\text {Nd(t) }}$ & $\mathrm{U}-\mathrm{Pb}(\mathrm{Ma})$ \\
\hline $\mathrm{Rb}-01$ & Gabro & $+5,8$ & na & na & 1,75 & $+1,9$ & \\
$\mathrm{Rb}-02$ & Gabro & $+5,4$ & -88 & $+3,8$ & 1,80 & $+1,2$ & \\
$\mathrm{Rb}-04$ & Gabro & $+5,6$ & na & na & 1,17 & $+8,9$ & $1469 \pm 31$ \\
$\mathrm{Rb}-06$ & Qz-monzogabro & $+8,3$ & -83 & $+0,7$ & 1,81 & 0,0 & \\
$\mathrm{Rb}-07$ & Qz-monzogabro & $+7,3$ & -85 & $+1,2$ & 1,82 & $+1,2$ & \\
$\mathrm{Rb}-08$ & Granito & $+9,0$ & -90 & $+2,8$ & 1,84 & $-0,3$ & \\
$\mathrm{Rb}-09$ & Granito & $+8,9$ & -85 & $+6,1$ & 1,85 & $-0,5$ & \\
$\mathrm{Rb}-12$ & Granito & $+8,7$ & -92 & $+5,2$ & 1,89 & $-0,4$ & $1423 \pm 02$ \\
\hline
\end{tabular}

* Os resultados de isótopos de 0 são apresentados normalizados pelo SMOW. ** 0 s resultados de isótopos de $\mathrm{H}$ são apresentados normalizados pelo SMOW. $\star \star *$ Os resultados de isótopos de $S$ são apresentados normalizados pela troilita do Canyon Diablo (CDT). na = não analisado. isotópica da fonte, uma vez que não há evidências de interação entre o magma gerador das rochas e as encaixantes regionais representadas pelas rochas do Terreno Jauru anteriormente apresentadas. Os valores de $\delta^{34} S$ obtidos devem indicar baixa fugacidade de oxigênio, o que permitiu a deposição de sulfeto de ferro (pirita), apesar de se observar poucos grãos de magnetitas em estudos petrográficos. Nos estudos envolvendo isótopos de $\mathrm{S}$, os valores de $\delta^{34} \mathrm{~S}$ em sulfetos de meteoritos são comumente utilizados para a normalização e definidos como $\delta^{34} S=0 \pm 0,5 \%$ o (Ohmoto, 1984; Cameron, 1986; Taylor, 1987; Chaussidon et al., 1989), já que suas composições são interpretadas como equivalentes à composição da Terra anteriormente aos processos de diferenciação e formação de crosta continental. Desta forma, quanto mais próximo o valor de $\delta^{34} S$ obtido na amostra for de zero, maior influência do manto na gênese da rocha. A única amostra básica da Suíte Intrusiva Rio Branco analisada (RB-02) apresentou valor de $\delta^{34} S$ de $+3,8 \%$ o sugerindo uma fonte manto-derivada

para a sua origem, coerente com o valor de $\boldsymbol{E}_{\mathrm{Nd}}(+1,2)$. Já amostras Rb-12 e Rb-09 apresentaram valores de $\delta^{34} S$ de $+6,1$ e $+5,2 \%$, respectivamente, sugerindo um componente crustal na gênese de seus protólitos, em concordância para os valores médios de $\delta^{34} \mathrm{~S}=7,0 \%$ para a crosta terrestre, segundo Chaussidon et al. (1989) e Taylor (1974). Entretanto as amostras Rb-06 e Rb-07 apresentaram os menores valores de $\delta^{34} S(+0,7 \%$ e $+1,7 \%$, respectivamente), indicando derivação mantélica, contrapondo os valores de $\delta^{18} \mathrm{O}(+8,3 \%$ e $+7,3 \%$,

\begin{tabular}{|c|c|c|c|c|}
\hline & 0 & +5 & +10 & +15 \\
\hline $\begin{array}{l}\text { Suíte Intrusiva } \\
\text { Rio Branco }\end{array}$ & & & & \\
\hline $\begin{array}{l}\text { RB-01 } \\
\text { gabro }\end{array}$ & & $\star$ & & \\
\hline $\begin{array}{l}\mathrm{Rb}-02 \\
\text { gabro }\end{array}$ & & $\%$ & & \\
\hline $\begin{array}{l}\mathrm{Rb}-04 \\
\text { gabro }\end{array}$ & & * & & \\
\hline $\begin{array}{l}\text { Rb-06 } \\
\text { qz-monzogabro }\end{array}$ & & & * & \\
\hline $\begin{array}{l}\text { Rb-07 } \\
\text { qz-monzogabro }\end{array}$ & & & * & \\
\hline $\begin{array}{l}\mathrm{Rb}-08 \\
\text { granito }\end{array}$ & & & * & \\
\hline $\begin{array}{l}\mathrm{Rb}-09 \\
\text { granito }\end{array}$ & & & * & \\
\hline $\begin{array}{l}\mathrm{Rb}-12 \\
\text { granito }\end{array}$ & & & * & \\
\hline
\end{tabular}

Figura 3 - Diagrama com os dados de $\delta^{18} 0$ da Suíte Intrusiva Rio Branco. 


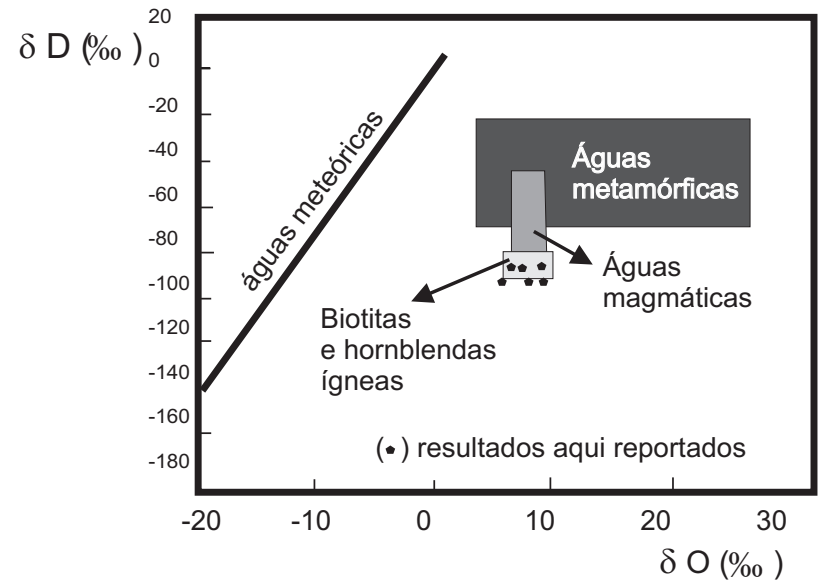

Figura 4 - Diagrama $\delta$ D versus $\delta^{18} 0$ para as rochas da Suíte Intrusiva Rio Branco mostrando as composições de diferentes tipos de águas naturais descritas por Taylor (1974). Os resultados das amostras analisadas são coerentes com assinaturas de águas de origem magmática. A dimensão dos pontos lançados no diagrama não representam erro analítico.

respectivamente) que indicam uma maior influência crustal na origem destas rochas.

\section{TERRENO PONTES E LACERDA}

Seis amostras de rochas granitóides da Suíte Santa Helena foram analisadas para isótopos estáveis de oxigênio. As amostras selecionadas foram o Tonalito Lavrinha (97-113), o Gnaisse Granodiorítico (97-108), o Gnaisse Santa Helena (97-115), o Granito Serrana (97-116) e o Granodiorito Comodoro (97-127). Os resultados de ${ }^{18} \mathrm{O} /{ }^{16} \mathrm{O}$ foram normalizados pelo standard SMOW e são apresentados na Tabela 3. A localização das amostras encontra-se na Figura 1B.

Os resultados da Tabela 3 indicam uma variação entre $+4,4 \%$ e e $+8,9 \%$ p para as rochas da Suíte Santa Helena. A partir dos resultados obtidos pode-se dividir as amostras em dois grupos com valores de $\delta^{18} \mathrm{O}$ distintos (Figura 5): o primeiro grupo com valores de $\delta^{18} \mathrm{O}=+4,4 \%$ (amostras 97-113, 97115 e 97-116) e o segundo grupo (amostras 97-108 e 97-117) com $\delta^{18} \mathrm{O}$ de $+8,3 \%$ e $8,9 \%$, respectivamente.

Os resultados de $\delta^{18} \mathrm{O}$ do primeiro grupo $(+4,4 \% 0)$ são inferiores ao valor estimado para o manto $\left(\delta^{18} \mathrm{O}=5,75 \% 0 \pm 0,3\right.$ segundo Taylor, 1980). Estudos petrográficos nas rochas deste

Tabela 3 - Valores de $\delta^{18} 0$ para as rochas granitóides da Suíte Santa Helena. Valores de U-Pb e Sm-Nd obtidos em Tassinari et al. (2000).

\begin{tabular}{llcccc}
\hline Amostra & Descrição & $\delta^{18} 0(\%)$ & $\varepsilon_{\text {Nd }}$ & $\mathrm{T}_{\text {DM }}(\mathrm{Ga})$ & $\mathrm{U}-\mathrm{Pb}(\mathrm{Ma})$ \\
\hline $97-113$ & Tonalito Lavrinha & $+4,4$ & $+3,8$ & 1,53 & $1464 \pm 25$ \\
$97-108$ & Granodiorito Guaporé & $+8,9$ & $+3,4$ & 1,54 & $1435 \pm 22$ \\
$97-115$ & Gnaisse Santa Helena & $+4,4$ & $+3,1$ & 1,62 & $1433 \pm 06$ \\
$97-116$ & Granito Serrana & $+4,4$ & $+2,8$ & 1,57 & $1424 \pm 11$ \\
$97-127$ & Granodiorito Comodoro & $+8,3$ & - & - & - \\
\hline
\end{tabular}

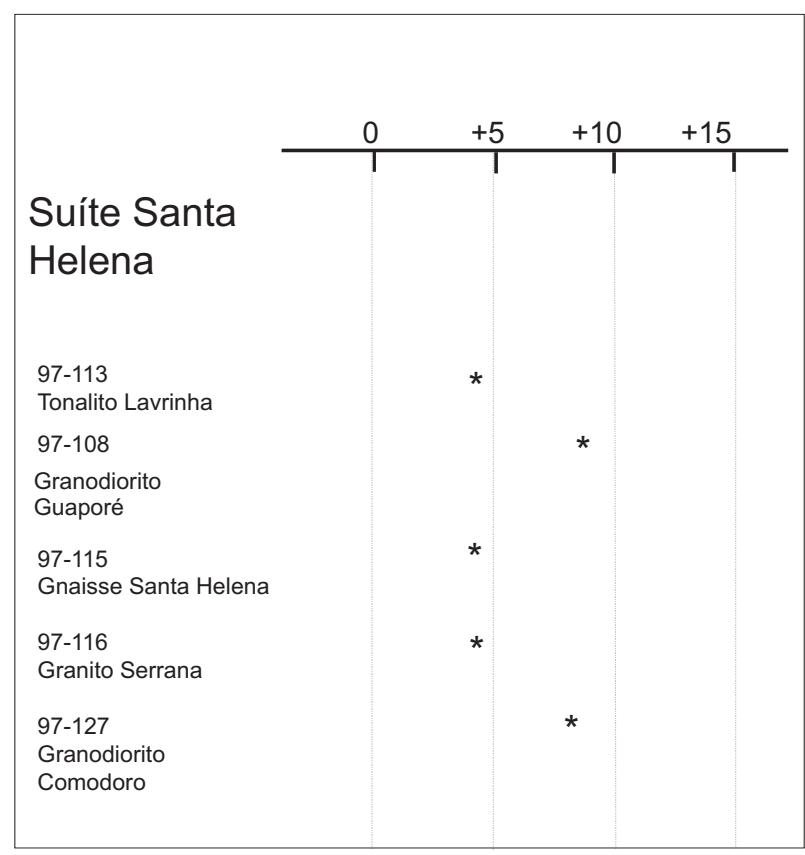

Figura 5 - Resultados de isótopos de $\delta^{18} 0$ para as rochas da Suíte Santa Helena.

grupo não indicaram alteração que promovesse a diminuição dos valores de $\delta^{18} \mathrm{O}$ como a interação com águas meteóricas. Entretanto pode ter havido interação destas rochas com soluçôes hidrotermais com baixos valores de $\delta^{18} \mathrm{O}$ ainda na fase subsolidus da cristalização. Caso semelhante foi observado por Taylor (1988) em rochas graníticas nas Ilhas Seychelles com valores de $\delta^{18} \mathrm{O}$ de até $-5,0 \%$. $\mathrm{O}$ autor ressalta ainda que no exemplo estudado não teria ocorrido modificação dos valores de $\varepsilon_{\mathrm{Nd}}$. As idades $\mathrm{U}-\mathrm{Pb}$ (entre 1,46 e 1,42 Ga) e as $\mathrm{T}_{\mathrm{DM}}$ entre 1,62 e $1,53 \mathrm{Ga}\left(\mathrm{com} \varepsilon_{\mathrm{Nd}}\right.$ entre $+3,8$ e $\left.+2,8\right)$ reforçam as características juvenis para as rochas deste grupo da Suíte Santa Helena.

Os resultados de $\delta^{18} \mathrm{O}$ para as amostras do segundo grupo da Suíte Santa Helena (Granodiorito Guaporé com $+8,9 \%$ e Granodiorito Comodoro com $+8,3 \%$ ) são coerentes com valores de $\delta^{18} \mathrm{O}$ característicos de rochas crustais. No entanto, a idade U-Pb para o Granodiorito Guaporé é de 1,44 Ga, $\mathrm{T}_{\mathrm{DM}}$ de 1,54 e $\varepsilon_{\mathrm{Nd}} \mathrm{de}+3,4$ o que indica características juvenis para este grupo. Em adição, as rochas da Suíte Santa Helena mostram uma ampla variação nas composições isotópicas de $\mathrm{Pb}{ }^{206} \mathrm{~Pb} /{ }^{204} \mathrm{~Pb}$ entre 20,044 e 16,$960 ;{ }^{207} \mathrm{~Pb} /{ }^{204} \mathrm{~Pb}$ entre 15,789 e 15,$409 ; \mathrm{e}^{208} \mathrm{~Pb} / 204 \mathrm{~Pb}$ entre 37,707 e 35,875 ) que foram interpretadas como decorrentes da re-homogeneização durante o evento de cavalgamento Aguapeí (Geraldes et al., 
2001). As variações nos valores de $\delta^{18} \mathrm{O}$ aqui apresentados podem ter sido decorrentes desta re-homogeneização isotópica no final do Mesoproterozóico.

\section{CONCLUSÕES}

Os dados isotópicos de $\mathrm{O}, \mathrm{H}$ e $\mathrm{S}$ mostram-se úteis na determinação de fontes de rochas ígneas, e apresentam importante contribuição ao entendimento da evolução geológica de processos magmáticos. No SW do Craton Amazônico foram estudas rochas dos terrenos Jauru e Pontes e Lacerda. No primeiro, as unidades amostradas foram o greenstone belt Alto Jauru, a Suíte Cachoeirinha e a Suíte Intrusiva Rio Branco. No terreno Pontes e Lacerda a unidade estudada foi a Suíte Santa Helena.

Os dados isotópicos de $\delta^{18} \mathrm{O}$ das rochas granitóides do greenstone belt Alto Jauru apresentaram valores entre $+6,3 \%$ e $+6,6 \%$ o. Estes valores são coerentes com os dados de $\mathrm{Sm}-\mathrm{Nd}$ para as mesmas amostras ( $\mathrm{T}_{\mathrm{DM}}$ entre 1,93 Ga e 1,77 Ga e $\varepsilon_{\mathrm{Nd}(\mathrm{t})}$ entre $+2,6$ e $+2,2$ ), que são característicos de suítes calcioalcalinas geradas em arcos magmáticos. As rochas calcioalcalinas geradas em ambiente de arco magmático usualmente apresentam valores de $\delta^{18} \mathrm{O}$ entre $+6,5 \mathrm{e}+8,0 \%$, ao passo que rochas supracrustais usualmente apresentam valores de $\delta^{18} \mathrm{O}$ entre $+6,0$ e $+18,0 \%$ o segundo Taylor (1988). A suíte Cachoeirinha apresentou valores de $\delta^{18} \mathrm{O}$ entre $+6,3 \%$ o e $+9,0 \%$ e valores de $\mathrm{T}_{\mathrm{DM}}$ entre $1,78 \mathrm{Ga}$ e $1,75 \mathrm{Ga}$, com $\varepsilon_{\mathrm{Nd}(\mathrm{t})}$ variando de $+0,5 \mathrm{a}+1,7$. Com exceção do valor obtido para o Granodiorito Água Clara (+9,0\%), os dados permitem concluir que os granitóides analisados no Terreno Jauru foram derivados de magmas juvenis e não apresentam indícios de processos ocorridos a baixas temperaturas. Rochas cujas fontes magmáticas apresentam valores de $\delta^{18} \mathrm{O}$ maiores do que $+8 \%$ o requerem o envolvimento de algum material que em certo período de tempo no passado tenha residido próximo da superfície.

A Suíte Intrusiva Rio Branco tem caráter bimodal, tendo sido formada em ambiente intra-cratônico durante o Mesoproterozóico (1,47-1,42 Ga). As rochas félsicas desta suíte apresentaram características químicas de granitos rapakivi e valores de $\delta^{18} \mathrm{O}$ de $+8,7 \%$ a $+9,0 \%$. Para as rochas básicas os valores variaram de $+5,4 \% 0 \mathrm{a}+5,8 \%$; as rochas intermediárias apresentaram valores de $\delta^{18} \mathrm{O}$ de $+7,3 \%$ a $+8,3 \%$. Os valores $\delta^{18} \mathrm{O}$ do grupo de rochas básicas são compatíveis com uma fonte mantélica e as rochas félsicas apresentaram valores de $\delta^{18} \mathrm{O}$ compatíveis com origem crustal. Os valores de $\delta^{18} \mathrm{O}$ intermediários podem indicar fontes híbridas, resultado de processos como assimilação, interação magma-crosta ou metassomatismo (contaminação) do manto através do retorno de sedimentos em zonas de subducção. Os dados aqui apresentados não permitem discriminar entre estes vários processos qual foi o predominante.

Os resultados das análises de isótopos estáveis de $\mathrm{H}$ em rocha total (de $-83 \%$ a $-92 \%$ ) da Suíte Intrusiva Rio Branco, combinados com isótopos de $\delta^{18} \mathrm{O}$, indicam uma origem co-magmática para as soluçôes hidrotermais que provocaram as variaçôes isotópicas nas rochas desta unidade. Estes resultados, por sua vez, não suportam a hipótese de participação de águas meteóricas ou metamórficas nas soluçôes hidrotermais formadoras das amostras estudadas. Os resultados em sulfetos de isótopos estáveis de $S$ para as rochas básicas e intermediárias apresentaram valores de $\delta^{34} \mathrm{~S}$ coerentes com uma fonte mantélica (entre $+0,7 \%$ e $+3,8 \%$ ) enquanto os valores de $\delta^{34} S$ (entre $+5,2 \%$ e e $+6,1 \%$ ) obtidos nas rochas félsicas sugerem participação crustal na sua gênese. Esta contribuiçãao pode ter ocorrido na forma de assimilação, interação magma-crosta ou contaminação do manto através do retorno de sedimentos em zonas de subducção. Os resultados aqui apresentados não permitem discriminar entre estes vários processos. A conjunção dos dados de isótopos de $\mathrm{O}, \mathrm{H}$ e S e os dados de isótopos radiogênicos (U-Pb e $\mathrm{Sm}-\mathrm{Nd}$ ) publicados na literatura reforçam a interpretação que a Suíte Intrusiva Rio Branco representa parte de uma associação bimodal não cogenética, em que as rochas básicas foram derivadas do manto, enquanto as rochas félsicas se formaram na base da crosta.

Os dados isotópicos de O das rochas da Suíte Santa Helena (entre $+4,4 \%$ o e $+8,9 \%$ ), que ocorrem próximo da cidade de Pontes e Lacerda, mostram uma maior complexidade de resultados e interpretações em relação aos resultados de isótopos estáveis obtidos em parte das rochas graníticas da região de Jauru-Araputanga. A partir dos resultados obtidos pode-se dividir as amostras em dois grupos com valores de $\delta^{18} \mathrm{O}$ distintos: o primeiro grupo, com valores ao redor de $\delta^{18} \mathrm{O}$ $=+4,4 \%$ o, define uma variação inferior ao valor estimado para o manto $\left(\delta^{180}=5,0 \%\right.$ o), provavelmente resultado da interação destas rochas com soluções hidrotermais com baixos valores de $\delta^{18} \mathrm{O}$ ainda na fase subsolidus da cristalização. Estes valores de $\delta^{18} \mathrm{O}$ combinados com as idades U-Pb (entre 1,46 e 1,42 $\mathrm{Ga})$ e as $\mathrm{T}_{\mathrm{DM}}$ entre 1,62 e $1,53 \mathrm{Ga}\left(\operatorname{com} \varepsilon_{\mathrm{Nd}}\right.$ entre $+3,8$ e $+2,8)$ reforçam as características juvenis para as rochas deste grupo.

Os resultados de $\delta^{18} \mathrm{O}$ para as amostras do segundo grupo da Suíte Santa Helena (entre $+8,9 \%$ e e $+8,3 \%$ ) são coerentes com valores de $\delta^{18} \mathrm{O}$ característicos de rochas crustais. Em adição, as rochas da Suíte Santa Helena mostram uma ampla variação nas composições isotópicas de $\mathrm{Pb}\left({ }^{206} \mathrm{~Pb} /{ }^{204} \mathrm{~Pb}\right.$ entre 20,044 e 16,$960 ;{ }^{207} \mathrm{~Pb} /{ }^{204} \mathrm{~Pb}$ entre 15,789 e 15,409 ; e 
${ }^{208} \mathrm{~Pb} /{ }^{204} \mathrm{~Pb}$ entre 37,707 e 35,875$)$ que foram interpretadas como decorrentes da re-homogeneização durante o evento de cavalgamento Aguapeí. As variações nos valores de $\delta^{18} \mathrm{O}$ aqui apresentados podem ter sido decorrentes da percolação de soluções hidrotermais no final do Mesoproterozóico.

\section{AGRADECIMENTOS}

Este trabalho foi financiado pela FAPESP, Processo 9604819-7 para M. C. Geraldes, Processo FAPESP 96-1207-1 para W. Teixeira. O trabalho é uma contribuição para o Projeto UNESCO IGCP 510 - A-Type Granites and Related Rocks Through Time.

\section{BIBLIOGRAFIA CITADA}

Agrinier, P.; Javoy, M.; Smith, D.C.; Pineau, F. 1985. Carbon and oxygen isotopes in eclogites, amphibolites, veins and marbles from the western gneiss region, Norway. Chemical Geology, 52:145-162.

Baumgartner, L.P.; Rumble, D. 1988. Transport of stable isotopes I: Development of kinetic continuum theory for stable isotope transport. Contributions to Mineralogy and Petrology, 98:417430.

Cameron, E.M. 1986. Sulphate and sulphate reduction in early Precambrian oceans. Nature, 296:145-148.

Chaussidon, M.; Albarede, F.; Sheppard, S.M.F. 1989. Sulfur isotope variations in the mantle from ion microprobe analysis of micro-sulphide inclusions. Earth Planetary Science Letters, 92:144-156.

Clayton, N.R.; Mayeda, T. 1963. The use of bromide pentafluoride in the extraction of oxygen from oxides and silicates for isotope analysis. Geochimica et Cosmochimica Acta, 27:43-52.

Clayton, N.R.; Goldsmith, J.R.; Mayeda, T. 1989. Oxygen isotope fractionation in quartz, albite, anorthite and calcite. Geochimica et Cosmochimica Acta, 53:725-733.

Claypool, G.E.; Holser; I.R.; Sakai, H.; Zak, I. 1980. The age curve of sulfur and oxygen isotopes in marine sulfate and their mutual interpretation. Chemical Geology, 28:199-259.

Eiler, J.M.; Baumgartner, L.P.; Valley, J.W. 1992. Intercrystalline stable isotope diffusion: a fast grain boundary model. Contributions to Mineralogy and Petrology, 112:543-557.

Forester, W.R. and Taylor, H.P. 1977. ${ }^{18} \mathrm{O} /{ }^{16} \mathrm{O}, \mathrm{D} / \mathrm{H}$ and ${ }^{13} \mathrm{C} /{ }^{12} \mathrm{C}$ studies of the Tertiary igneous complex of Skye, Scotland. American Journal of Science, 277:136-177.

Geraldes, M. C.; Teixeira, W.; Heilbron, M. 2004. Lithospheric versus asthenospheric source of the SW Amazonian craton A-types granites: the role of the Paleo and Mesoproterozoic accretionary belts for their coeval continental suítes. Episodes, 27(3):185-189.

Geraldes, M.C.; Van Schmus, W.R., Condie, K.C., Bell, S., Teixeira, W.; Babinski, M. 2001. Proterozoic Geologic Evolution of the SW part of the Amazonian Craton in Mato Grosso State, Brazil. Precambrian Research, 111:91-128.
Golding, S.D.; Wilson, A.F. 1983. Geochemical and stable isotope studies of the no 4 lode, Kalgoorlie, Western Australia. Economic Geology, 78: 438-450.

Goodz, M.D.; Watkinson, D.H.; Smejkal, V., Pertold, Z. 1986. Sulfur-isotope geochemistry of silver-sulpharsenide vein mineralization, Cobalt, Ontario. Canadian Journal of Earth Science, 23:1551-1567.

Harris, C.; Faure, K.; Diamond, R.E.; Scheepers, R. 1997. Oxygen and Hydrogen isotope geochemistry of S- and I-type granitoids: the Cape Granite suite, South África. Chemical Geology, 143:95114.

Iyer, S.S.; Hoefs, J.; Krouse, H.R. 1992. Sulfur and lead isotope geochemistry of galenas from Bambuí Group, Minas Gerais, Brazil -- implications for ore genesis. Economic Geology, 87: 437-444.

Kanehiro, K.; Yui, S.; Sakay, H.; Sasaki, A. 1973. Sulphide globules and sulphur isotope ratios in the abyssal tholeiite from the Mid-Atlantic Ridge near $30^{\circ} \mathrm{N}$ latitude. Geochemical Journal, 7:89-96.

Monteiro, H.; Macedo, P.M. de; Silva, M.D. da; Moraes, A.A. de; Marcheto, C.M.L. 1986. O greenstone belt do Alto Jauru. Anais do XXXIV Congresso Brasileiro de Geologia. vol. 2: p.630-646.

Martarelli, L.; Ferrini, V.; Mais, U. 1995. Trace-element evidence for the genesis of the pyrite vein deposit of Campiano (southern Tuscany, Italy). Contributions to Mineralogy and Petrology, 64:349-366.

Menezes, R. G. de; Lopes, I.; Bezerra, J.R.L. 1993. Folha Pontes e Lacerda 1:100.000. Carta Geológica e Texto Explicativo. Programa de Levantamentos Básicos. CPRM-DNPM. 176pp.

Nabelek, P. 1991. Stable isotope monitors. Contact Metamorphism. In: D.M. Keirrich (Ed). Reviews in Mineralogy, 26:211-435.

Nabelek, P.I.; O’Neil, J.R.; Papike, J.J. 1983. Vapor phase exsolution as a controlling factor in hydrogen isotope variation in granitic rocks: the Notch Peak granite stock, Utah. Earth and Planetary Science Letters, 66:137-150.

Ohmoto, H. 1984. Stable isotope geochemistry of ore deposit. In: Valley JW, Taylor, HP, O’Neil JR (Eds). Stable Isotopes in high temperature geological process. Reviews in Mineralogy, 16: 491-570.

O’Neil, J.R. 1979. Stable isotopes Geochemistry of rocks and minerals. In: R. Jager; J.C. Hunziker (Eds). Lectures in Isotope Geology. Springer-Verlag, Berlim. 1979. p.127-138.

O'Neil, J.R.; Taylor, H.P. 1967. The oxygen isotope and cation exchange chemistry of feldspars. American Mineralogist, 52:14141437.

O’Neil, J.R.; Shaw, S.; Flood, R.H. 1977. Oxygen and Hydrogen isotope composition as indicators of granite genesis in the New England Batholith, Austrália. Contributions to Mineralogy and Petrology, 62:313-328.

Palin, J.M.; Epstein, S.; Stolper, E.M. 1996. Oxygen isotope partitioning between rhyolitic glass/melt and $\mathrm{CO}_{2}$ : an 
experimental study at $550-950^{\circ} \mathrm{C}$ and 1 bar. Geochimica et Cosmochimica Acta. 60(11):1963-1973.

Roeder, E. 1992. Fluid inclusion evidence for immiscibility in magmatic differentiation. Geochimica et Cosmochimica Acta, 56:5-20.

Rollinson, H. 1993 Using geochemical data: evaluation, presentation, interpretation. Longman Scientific. Technical. England. 352pp.

Ryerson, F.J.; McKeegan, K.D. 1994. Determination of oxygen self-diffusion in akermanite, anorthite, diopside and spinel: Implications for oxygen isotopic anomalies and the thermal histories of Ca-Al-rich inclusions. Geochimica et Cosmochimica Acta, 58(17):3713-3734.

Ruiz, A. S.; Geraldes, M. C.; Matos, J.; Teixeira, W.; Van Schmus, W.R.; Schmitt, R.S. 2004. The 1590-1520 Ma Cachoeirinha magmatic arc and its tectonic implications for the Mesoproterozoic SW Amazonian craton crustal evolution. Anais da Academia Brasileira de Ciências, 76(4):808-824.

Saes, G.S.; Leite, J.A.D.; Weska, R.K. 1984. Geologia da Folha Jauru (SD-21-Y-C-III): Uma Síntese dos Conhecimentos. Anais do XXXIII Congresso Brasileiro de Geologia. vol. 5: p.2193-2204.

Sial, A.N. 1984. Padrão regional de isótopos de oxigênio em granitóides do espaço Cachoeirinha, Nordeste do Brasil. Anais do XXXIII Congresso Brasileiro de Geologia. Rio de Janeiro, 1984. p. 2710-2719.

Sial, A.N.; Ferreira, V.P 1990. Granitoids in northeastern Brazil: Oxygen and sulfur isotopes compositions and depths of emplacement. Journal of South American Earth Sciences, 3(2/3):103-112.

Sial, A.N.; Toselli, A.J.; Ferreira, V.P.; Rossi de Toselli e Fallick, A.E. 1996. Granitóides com epidoto magmático da Província de Borborema, NE do Brasil e das Serras Pampeanas, NW da Argentina. Boletim do Instituto de Geociências, 5:51-53.

Souza, A.E.P.; Hildred, P.R. 1980. Contribuição ao estudo da geologia do Grupo Aguapeí, Mato Grosso. Anais do XXXI Congresso Brasileiro de Geologia. Vol. 2: p. 587-598.

Spooner E.; Wood P.; Burrows, D., Thomas, A.; Noble, S. 1985. Geological fluid inclusion and isotope (C and S) studies of gold-quartz-cabonate-pyrite-scheelite vein mineralization and intrusion hosted $\mathrm{Cu}-\mathrm{Au}-\mathrm{Mo}$ mineralization in the HollingerMcIntyre system, Timmings Ont. Ontario Geological Survey Miscelaneous Paper, 127:229-246.

Tassinari, C.G., Bettencourt, J.S., Geraldes, M.C., Macambira, M.J.B., and Lafon, J.M., 2000. The Amazon craton. In: Cordani, U.; Milani, E.J.; Thomaz Filho, A.; Campos, D.A. (Eds). Tectonic evolution of South America. XXXI International Geological Congress, Rio de Janeiro, Brazil, 41-95.

Taylor, B.E. 1987. Stable isotope geochemistry of ore-forming fluids. In: Kyser T, Kurtis T (Eds). Stable isotope geochemistry of low temperature process. Canadian Mineralogist. Short Course Handbook, 13: 337-445.

Taylor, H.P. 1974. The application of oxygen and hydrogen isotope studies to problems of hydrothermal alteration and ore deposition. Economic Geology, 843-883.

Taylor, H.P. 1980. The effects of assimilation of country rocks by magmas on ${ }^{18} \mathrm{O} /{ }^{16} \mathrm{O}$ and ${ }^{87} \mathrm{Sr} /{ }^{86} \mathrm{Sr}$ systematics in igneous rocks. Earth and Planetary Science Letters, 47: 243-254.

Taylor, H.P.1988. Oxygen, hydrogen, and strontium isotope constraints on the origin of granites. Transactions of the Royal Society of Edinburg: Earth Sciences, 79: 317-338.

Van Wyck, N.; Valley, J.W.; Austrheim, H. 1966. Oxygen and carbon isotopic constraints on the development of eclogites, Holsoy, Norway. Lithos 38: 129-145.

Vanko, D.A., Griffith, J. D. and Erickson, C.L. 1992. Calcium-rich brines and other hydrothermal fluid inclusions from plutonic rocks, Oceanografer Tranform, mid-Atlantic Ridge. Geochimica et Cosmochimica Acta, 56: 35-47.

Wenner, D.B. and Taylor, H.P. 1976. Oxygen and hydrogen isotope studies of a Precambrian granite-rhyolite terrane, St. François Mointais, southeastern Missouri. Geological Society of America Bulletin, 87: 1587-1598.

Recebido em 04/04/2006

Aceito em 07/12/2008 
